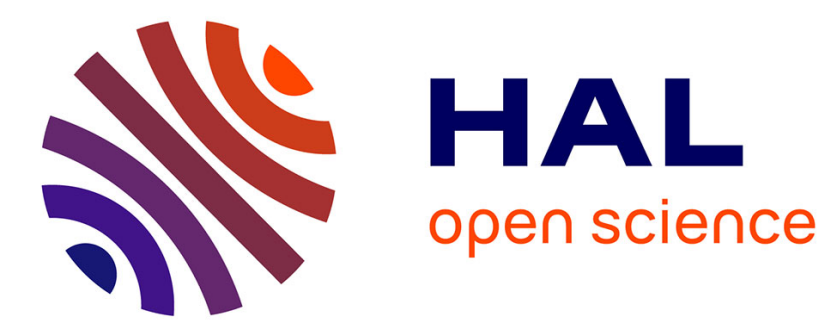

\title{
Les ateliers du spectateur, fabriques du sensible Philippe Guisgand
}

\section{To cite this version:}

Philippe Guisgand. Les ateliers du spectateur, fabriques du sensible. Quaderni, 2014, 83, pp.59-72. 10.4000/quaderni.764 . hal-02168493

\section{HAL Id: hal-02168493 https://hal.science/hal-02168493}

Submitted on 4 Jul 2019

HAL is a multi-disciplinary open access archive for the deposit and dissemination of scientific research documents, whether they are published or not. The documents may come from teaching and research institutions in France or abroad, or from public or private research centers.
L'archive ouverte pluridisciplinaire HAL, est destinée au dépôt et à la diffusion de documents scientifiques de niveau recherche, publiés ou non, émanant des établissements d'enseignement et de recherche français ou étrangers, des laboratoires publics ou privés. 


\title{
Les ateliers du spectateur, fabriques du sensible
}

\section{Audience workshops : trademark of the sensitive}

\author{
Philippe Guisgand
}

\section{Introduction}

L'activité régulière de transmission liée à la profession d'enseignant chercheur pose la question de la place de la vulgarisation des connaissances. D'autant qu'en France, Patrick Germain-Thomas le souligne dans son introduction au présent dossier, les ressources permettant d'assoire une honnête culture chorégraphique pour le plus grand nombre demeurent rares. Ce constat contribue à renforcer une conviction : adapté à un auditoire plus large, les éléments de cette culture dispensée au sein de l'Université peut dépasser la petite communauté de mes étudiants. C'est pourquoi - comme nombre de collègues, j'ai travaillé à des contributions ou des ouvrages destinés initialement aux spécialistes mais qui, par leur format, leur composition ou leur accessibilité, ambitionnent d'agrandir ce premier cercle. Progressivement, cette activité s'est déclinée sous la forme d'entretiens dans des journaux spécialisés ou de conférences débordant le cadre confidentiel des communications scientifiques ${ }^{1}$. Puis les demandes émanant des structures culturelles de diffusion l'ont encore davantage infléchie vers l'oral : conférences, tables rondes, introductions à des répétitions publiques, regards critiques ou débats d'après spectacle sont devenues mes modalités d'intervention autours des œuvres chorégraphiques et performatives les plus fréquentes. Sollicité régulièrement, je me suis aperçu qu'au fil du temps, je répondais plus favorablement à certaines d'entre elles et en refusait d'autres plus systématiquement. Expliciter ces choix intuitifs m'a amené à penser plus sérieusement ce pan d'une activité rarement reconnue sur le plan scientifique. En effet, son déni - au motif que la vulgarisation serait une pensée et/ou une écriture "au rabais" - est encore fréquent. Une telle posture sert d'excuse aux chercheurs qui ne s'y arrêtent pas ou autorise la prise de distance quelque peu condescendante des mêmes à l'égard de ceux qui trouvent cette mission intéressante, voire même valorisante, dans l'exercice du métier... Au point d'en faire, dans mon cas, un axe de travail érigeant les ateliers dont je vais parler en véritable laboratoire de la réception spectatorielle. Contextualiser ces interventions dans une perspective politique et culturelle m'a amené à en modifier la structure

\footnotetext{
${ }^{1}$ Elles sont majoritairement commandées par les structures culturelles de diffusion du spectacle vivant (Opéras, scènes nationales...), les lieux de formation (CNSMD, IEP, DRAC, ADIAM) ou les festivals culturels (Cité Philo, Monaco Dance Forum...).
} 
et mon rôle en leur sein. L'article qui suit, après avoir explicité ce contexte, puis analysé la démarche que j'y développe, s'attachera à rendre compte du déroulement de l'un de ces ateliers à travers une étude de cas concret.

\section{Un état du monde à infléchir collectivement}

Dans Le Partage du sensible, Jacques Rancière écrit :

J'appelle partage du sensible ce système d'évidences sensibles qui donne à voir en même temps l'existence d'un commun et les découpages qui y définissent les places et les parts respectives. Un partage du sensible fixe donc en même temps un commun partagé et des parts exclusives. Cette répartition des parts et des places se fonde sur un partage des espaces, des temps et des formes d'activité qui détermine la manière même dont un commun se prête à participation et dont les uns et les autres ont part à ce partage. ${ }^{2}$

Dans le domaine de la réception sensible du spectacle chorégraphique contemporain, cette situation de partage - au deux sens de communion et de répartition - trouve un double écho théorique : d'une part, dans le processus d'individuation forgé par Gilbert Simondon ${ }^{3}$ et que réclame Bernard Stiegler dans plusieurs de ses ouvrages et, d'autre part, dans une conception intimidante du rapport des spectateurs aux œuvres que les dispositifs culturels élaborent ou entretiennent - parfois à leur corps défendant. Je développerai successivement chacun de ces deux aspects.

Stiegler définit le concept d'individuation créé par Simondon comme un processus « à la fois psychique et collectif - où je et nous sont donc deux faces du même processus » ${ }^{4}$. C'est en faisant l'expérience d'une histoire collective, que le je finit par sentir l'appartenance à un nous qui serait une forme d'individu collectif. Autrement dit, elle est une modalité permettant à l'individu de devenir un et indivisible, à l'épreuve des relations que nécessite l'existence de toute activité collective. Mais pour Stiegler, la surconsommation des biens provoquée par l'ultralibéralisme des sociétés actuelles a pour effet de dissoudre ce je, non plus dans un nous signalant, notamment, un partage de valeur mais dans le on d'une masse gommant les relations du nous et qui supprime toute possibilité d'individuation. En devenant on, le je ne

\footnotetext{
2 Jacques Rancière, Le Partage du sensible, Paris, La Fabrique, 2000, p. 12.

${ }^{3}$ Gilbert Simondon, Du mode d'existence des objets techniques, Paris, Aubier, 1989. Pour Simondon, la biologie contemporaine fait la preuve qu'on ne peut concevoir l'individu comme un achevé possible mais, qu'au contraire, il n'y a que des organismes en individuation, c'est-à-dire en interaction et transformation constante avec leur milieu. L'individuation, comme devenir, serait l'essence de l'Homme.

4 Bernard Stiegler, «Politiques et industries de la culture dans les sociétés hyperindustrialisées » in Jean-Pierre Saez (ed.), Culture et société : un lien à recomposer, Toulouse, ed. de l'Attribut, 2008, p. 96 (l'auteur souligne).
} 
devient plus personne. Pour le philosophe, c'est l'industrie du mainstream, une réalité économique parfaitement décrite par Frédéric $\mathrm{Martel}^{5}$, qui est responsable de cet état de fait. Cette culture censée plaire à tout le monde a une fonction politique ancienne : elle permet de nier sans honte la réalité des disparités qui désormais sont, au sens propre, à nos portes. Mais ce marketing de contenu, issu de l'industrie du cinéma hollywoodien, opère également un conditionnement esthétique toujours plus coercitif : il y a plus de dix ans, Stiegler entrevoyait l'évolution d'une sphère «vidéo cliquable», enlaçant toujours plus les consciences et les synchronisant davantage aux objets temporels constituant les flux médiatiques. Dépassant ces prédictions, internet et la télévision sont désormais accessibles sur nos "téléphones" tactiles, c'est-à-dire partout et tout le temps ; les contenus sont profilés en fonctions des utilisateurs grâce aux signatures numériques laissées sur la toile au gré des navigations. Progressivement, en éliminant du temps disponible et donc incontrôlable, cette sphère élimine l'expérience esthétique inédite, «l'inattendu, autrement dit l'attente elle-même. » ${ }^{6}$

Ce conditionnement esthétique génère un relativisme culturel - un "tout se vaut pourvu qu'on l'aime" - qui noie les repères qualitatifs. On comprend mieux la logique marketing prescriptive fondée sur la psychologie des foules : allez voir tel film, puisque tout le monde l'a déjà vu... Ainsi s' " annihile la singularité des regards individuels ${ }^{7}$. Pour Stiegler, le film The Matrix (1999) en constitue une allégorie : les humains ne sont plus que des corps flottant dans des cuves dont l'énergie sert à alimenter des machines produisant des vies fictives simulées et synchronisées. Les individus deviennent incapables de lier leur je à d'autres dans la construction d'un nous qui constituerait l'espace d'une expérience esthétique partagée. Dans cette dynamique, le risque social de normopathie s'accroitt ${ }^{8}$. Par ailleurs, ce conditionnement provoque la haine des manifestations atypiques de l'art et de la culture. Cette culture bobo, qu'une petite minorité vit comme un enracinement/enrichissement, est ressentie par l'immense majorité comme un arrachement à ce qu'elle perçoit comme sa culture, commune puisque télévisuelle et légitime puisque partagée par le plus grand nombre.

Comment échapper à ce gouffre ? En liant esthétique et politique sans doute : « Ce qui atténue le relativisme, c'est la décantation progressive opérée par un débat inachevable, plus

\footnotetext{
${ }^{5}$ Frédéric Martel, Mainstream, enquête sur cette culture qui plait à tout le monde, Paris, Flammarion, 2010.

${ }^{6}$ Bernard Stiegler, «L'hyperindustrialisation de la culture et le temps des attrape-nigauds », in Internet all over, Art Press hors série, novembre 1999, p. 62.

${ }^{7}$ Bernard Stiegler, De la misère symbolique 1. L'époque hyperindustrielle, Paris, Galilée, 2004, p. 180.

${ }^{8}$ La normopathie désigne la tendance à se conformer excessivement à des normes sociales de comportement sans parvenir à exprimer sa propre subjectivité. La tuerie du lycée de Columbine en 1999 ou le cas de Richard Durn, tuant huit personnes à Nanterre en 2002 après avoir exprimé dans son journal un « irrépressible besoin d'exister », en sont des exemples. (Bernard Stiegler, Aimer, s'aimer, nous aimer. Du 11 septembre au 21 avril, Paris, Galilée, 2003, p. 14).
} 
que l'hypothétique établissement de critères universels et tranchants » écrit Rainer Rochlitz' Pour Stiegler aussi il faut réinstaurer un vivre ensemble où s'aimer c'est avant tout «aimer ensemble les choses (paysages, villes, objets, œuvres, langues etc.) ${ }^{10}$; où dialogue et partage s'appuieraient sur un fond esthétique commun. De ce fait, la hiérarchisation et la partition du sensible évoquées par Rancière doivent être réinterrogées. Il s'agira dès lors pour moi de promouvoir des modalités de relation aux oeuvres permettant de transformer le consommateur en amateur. Pour ce faire, il me paraît nécessaire de déverticaliser les relations entre acteurs du monde artistique et spectateurs. Cette une horizontalisation des échanges, cette déhiérarchisation des interlocuteurs constitue la condition sine qua non de rupture avec un accès à la culture artistique tendant à sacraliser l'artiste, sa parole et son œuvre. Plus largement, cette verticalité descendante s'observe à travers la valorisation de la posture experte et dans la relation bien souvent magistrale à "ce qu'il faudrait savoir". Alors que l'art contemporain consiste plutôt en un affranchissement des codes et significations traditionnels, le discours expert (critiques, artistes, acteurs du monde de l'art) provoque trop souvent une dépendance à ce qu'il faudrait comprendre, laissant le spectateur ordinaire désarmé et ne sachant plus que faire de ses sensations devenues inutiles. C'est pourquoi le sens doit aussi se fabriquer ensemble. Mais cette fabrication n'est possible qu'en se démarquant des réflexes de la culture experte au profit d'une égalité de convention où les rôles sociaux (création, contemplation, interprétation, théorisation) peuvent être discutés ou redistribués dans une démarche de compréhension de l'oeuvre. Cette dernière se révèle alors sous un double sens. Se comprendre, c'est découvrir les raisons pour lesquelles c'est ce monde, et pas un autre, qui nous apparaît. C'est aussi découvrir les raisons pour lesquelles nous nous sentons compris en lui. L'œuvre fait alors entendre le Nous communautaire dans cet enveloppement circulaire. Mais j'entends également par compréhension une forme de synthèse collective qui ne se déduit pas d'une simple interprétation ni d'une idiosyncrasie. Elle résulte au contraire de l'assemblage d'éléments qui, pris séparément (ou chez chacun) et indépendamment des autres, n'auraient pas le sens qu'ils produisent ensemble. Ce travail contemporain sur l'être ensemble est peut-être susceptible de renouveler un imaginaire collectif citoyen et/ou de (ré)activer une forme d'engagement politique.

\footnotetext{
${ }^{9}$ Rainer Rochlitz, Le Vif de la critique, tome 2 : Esthétique et philosophie de l'art, Bruxelles, La lettre volée, coll. «Essais », 2010, p. 336.

${ }^{10}$ Bernard Stiegler, De la misère symbolique 1. Op cit., p. 19.
} 


\section{Pour une démarche dévolutive}

Comment remplacer le pilotage masqué et le diktat uniformisant des industries culturelles - intimant d'aimer ceci ou de consommer cela ? Comment donner à chacun des moyens de forger sa propre subjectivité esthétique et contribuer à l'affinement de la construction de l'individu ? Ce souci du partage se cristallise autour d'un concept clé : la dévolution.

Dans le langage juridique banal, la dévolution désigne la transmission d'un bien d'une personne à une autre ; son usage historique précise qu'une entité supérieure, détentrice d'un pouvoir renonce à ses prérogatives en faveur d'une entité inférieure. Dans le champ didactique, elle consiste - par analogie - en un renoncement aux prérogatives du Maître. Elle oblige ainsi à l'adaptation des outils permettant à l'Elève de les utiliser. Elle éviterait de laisser le Maître dispenser les résultats avec parcimonie, préservant ainsi une partie de savoir nécessaire à son autorité sur l'Elève. Ce pari didactique fait écho aux analyses de Rancière sur l'assujettissement par l'explication. Dans «L'image intolérable » ${ }^{11}$, le philosophe dénonce la médiation des images violentes par la parole experte, entérinant l'idée que nous ne serions pas capables de les lire nous-mêmes et conduisant à un «abrutissement $»^{12}$. Dans Le Maître ignorant déjà, Rancière fustigeait le travail traditionnel du maître. A l'explication directe et écrite du fait que constitue le livre, se surajoute une explication orale du maître. Cette « logique de l'explication comporte ainsi le principe d'une régression à l'infini. » ${ }^{13}$ Or pour l'auteur, « expliquer quelque chose à quelqu'un, c'est d'abord lui démontrer qu'il ne peut pas l'apprendre par lui-même. ${ }^{14}$ Rancière étend ce modèle vertical d'assujettissement au social. L'explication, qu'incarne si bien aujourd'hui la culture de l'expertise, permet la mise en rang, la mise en ordre et la hiérarchisation. Et l'explication de devenir un instrument de domination.

Théorisée plus précisément dans le champ de la didactique des mathématiques, la dévolution désigne un ensemble de situations où l'action de l'élève n'est produite que par les nécessités du milieu et ses connaissances. Pourtant, on voit mal comment l'enseignant pourrait se soustraire à l'obligation d'enseigner et Brousseau - son promoteur - le reconnaît, cette situation génère un paradoxe. Tout ce que l'enseignant propose pour induire les comportements attendus prive l'élève d'une véritable compréhension autonome de

\footnotetext{
${ }_{11}^{11}$ Jacques Rancière, Le Spectateur émancipé, Paris, La fabrique, 2008, 93-114.

${ }^{12}$ Régis Debray plaidait lui aussi en son temps pour un enseignement scolaire de ce décryptage dans Vie et mort de l'image : une histoire du regard en Occident, Paris, Gallimard, coll. « Bibliothèque des idées », 1992.

${ }^{13}$ Jacques Rancière, Le Maître ignorant. Cinq leçons sur l'émancipation intellectuelle, Paris, 10/18, coll. « Fait et cause », [1987], 2004, p. 12.

${ }^{14}$ Idem, p. 15.
} 
l'apprentissage visé. Autrement dit, «Si le maître dit ce qu'il veut, il ne peut plus l'obtenir $»^{15}$. Par ailleurs, et pour séduisante qu'elle puisse paraître, cette solution didactique rencontre un écueil oublié mais majeur: l'engagement. En effet, il est aussi illusoire d'imaginer réveiller un sens citoyen en exhortant à l'indignation que d'espérer d'élèves qu'ils "se motivent" en fréquentant une école qui ne fait plus sens à leurs yeux et qui est obligatoire de surcroît. C'est pourquoi je crois la dévolution plus efficiente dans le cadre du débat esthétique. Volontaires, les participants à ces échanges ont librement choisi d'y adhérer. Les contenus ne sont que le résultat de la confrontation des perceptions et points de vue, que le chercheur organise et accompagne, sans obligation d'un résultat "juste" obligeant à élaborer un dispositif masqué amenant à l'objectif envisagé. Il n'y est jamais question de déverser, de façon confortable, un savoir condescendant ou intimidant frappé au sceau d'une "expertise" universitaire, mais d'y renoncer de façon que celui qui d'habitude écoute, prenne la parole.

La dévolution désignera donc ici l'ensemble évolutif des modalités de partage ou de confrontation des sensations, des mises en relation et des interprétations permettant de dépasser "l'art pour chacun" et l'assujettissement explicatif dénoncé par Rancière. Cet échange symétrique et communautaire devient une sorte de chœur qui va structurer sa manière de penser l'art au travail et en train de se faire. Dans le contexte d'un partage - et non d'une (ré)partition - du sensible propre aux arts, on voit comment un tel concept peut modifier le rapport à la parole experte et à la construction commune d'un sens. Je conçois donc la dévolution comme une «méthode émancipatrice» qui révèle « une intelligence à ellemême $»^{16}$. Et je rejoins pleinement Rancière dans l'objectif qu'il assigne à cette méthode prônée jadis par Jacotot ${ }^{17}$. L'exemple fourni par ce dernier permet à Rancière de critiquer le «mythe de la pédagogie », selon lequel un savoir aurait besoin d'être expliqué par le maître afin de pouvoir être compris par l'élève.). Il s'agit de dessiner «le modèle d'une société raisonnable où cela même qui est extérieur à la raison - la matière, les signes du langage - est traversé par la volonté raisonnable : celle de raconter et faire éprouver aux autres ce en quoi on est semblable. ${ }^{18}$ Je pense ces ateliers du spectateur comme une manifestation possible de ce fondement de la politique et comme un prétexte à une mise en commun du sensible débouchant sur une «fabrique du sensible [...], d'un monde sensible commun, d'un habitat

\footnotetext{
${ }^{15}$ Guy Brousseau, Théorie des situations didactiques, Grenoble, La Pensée sauvage, 1998, p. 73.

${ }^{16}$ Rainer Rochlitz, Le Vif de la critique, tome 2. Op. cit., p. 50.

${ }^{17}$ Dans son ouvrage Le Maître ignorant, Rancière s'intéresse à la méthode introduite par Joseph Jacotot au début $\mathrm{du} \mathrm{XIX}^{\circ}$ siècle et connue sous le nom de panécastique. Il en développe les principes tout en les comparant au système éducatif et social contemporain essentiellement fondé sur l'inégalité admise du savoir et de l'intelligence entre maître et élève

${ }^{18}$ Idem, p. 120.
} 
commun, par le tressage d'une pluralité d'activités humaines » ${ }^{19}$ où être, voir, dire et faire se recomposent dans cet être ensemble. La dévolution, telle que je l'envisage dans ce cadre, ne constitue pas une manière de se débarrasser de l'intercession entre l'œuvre et le spectateur, tantôt réclamée par l'une, tantôt souhaitée par l'autre. Au contraire, elle demande une discrimination et une réflexion sur l'usage du langage, des notions, des concepts, des formes de réception en amont de ces modalités de rencontre avec le geste dansé. Elle tentera donc d'envisager, d'une part, ce qui peut être différencié dans cette expérience commune et, d'autre part, ce qui peut être mis en commun de la somme des différences.

Cette confrontation/conciliation des points de vue paraît d'un grand intérêt pour opérer, en sens inverse, un mouvement vers la communauté. De prime abord, mettre en commun semble faire un obstacle au libre exercice des différences : d'où le succès médiatique de la formule «c'est mon choix » (entendons «c'est comme ça!») en lieu et place du débat. Or c'est bien plutôt le débat qui met en valeur la singularité d'un regard, le poids d'un argument, la richesse d'une contribution : rien n'oblige les "rassemblés" à se ressembler. Mais ce cercle peut prendre une consistance au travers de la qualité des échanges qui circulent à sa périphérie. Si je devais l'argumenter plus philosophiquement, je me tournerais vers Barbara Formis lorsqu'elle relève un effet de plan dans la relation touchant-touché chère à MerleauPonty. Peut-on « en même temps maintenir un niveau d'expérience d'ordre proprioceptif (me touchant moi-même) et un autre qui serait de l'ordre de la perception du monde (ma main touchant les choses) ${ }^{20}$ ? Cette conception du touché, transposée à l'expérience spectaculaire, propose l'idée de contact comme territoire commun. Ce déplacement va au-delà de la conception de Gérard Genette faisant de l'œuvre le point de rencontre entre intention de l'artiste et attention du lecteur (ou du spectateur). La simultanéité évoquée par Formis - du fait de n'être ni complètement tournée vers soi, ni complètement ouverte au monde -, engendre du "commun" à partager au sein d'une expérience intersubjective. Danse et regard ne s'élancent plus l'un vers l'autre dans un face-à-face que délimiterait la ligne scène-salle, mais s'interpénètrent pour créer des liens dans un espace partagé permettant, peut-être de faire “corps commun", un espace entre dans lequel le chorégraphique et son spectateur fusionnent en un même mouvement de danse et de pensée, et où la communauté esthétique se crée ${ }^{21}$.

\footnotetext{
${ }^{19}$ Jacques Rancière, Le Partage du sensible, op. cit., p. 66.

${ }^{20}$ Barbara Formis, « Toucher, bouger : la théorie somatique à l'épreuve de la vie », in Barbara Formis (ed.), Penser en corps. Soma-esthétique, art et philosophie, Paris, L’Harmattan, coll. « l'art en bref », 2009, p. 193.

${ }^{21}$ Pour Maldiney, l'art ménage un espace-temps de présence et de communication à tout : aux choses, aux êtres et à nous-mêmes (Regard, Parole, Espace, Lausanne, L'Age d'homme, 1973).
} 


\section{A propos de Mash Up (Maud Liardon, 2013)}

Les ateliers du spectateur, tels que je les ai conçus et les animent depuis 2009, pourraient figurer une mise à l'épreuve pratique de ce que Rancière désigne comme une communauté esthétique :

Les artistes, comme les chercheurs, construisent la scène où la manifestation et l'effet de leurs compétences sont exposés, rendus incertains dans les termes de l'idiome nouveau qui traduit une nouvelle aventure intellectuelle. L'effet de l'idiome ne peut être anticipé. Il demande des spectateurs qui jouent le rôle d'interprètes actifs, qui élaborent leur propre traduction pour s'approprier l' « histoire» et en faire leur propre histoire. Une communauté émancipée est une communauté de conteurs et de traducteurs. ${ }^{22}$

Ces ateliers créent pour moi cette forme de communauté éphémère autour des pratiques chorégraphiques scéniques. Ils s'ancrent dans une articulation perception-discussion, dans une expérience corpo-spectatorielle et s'en nourrissent pour produire de la polyphonie ou de la choralité. Enfin, soulignons que ces ateliers sont en phase avec l'évolution du modèle traditionnel de production : depuis une quinzaine d'année, en effet, certains chorégraphes se présentent volontiers comme initiateurs de groupements artistiques à vocations et visages multiples, plutôt qu'en qualité de directeurs de compagnies sous leurs propres noms : l'association remplace la compagnie (chez Boris Charmatz, Rachid Ouramdane ou Christian Rizzo). On n'a plus vraiment affaire à des structures produisant efficacement des pièces séduisantes, pour en exécuter la reproduction sur des plateaux. Ces groupes se vivent plutôt comme des communautés évolutives, rassemblées dans des espaces-temps privilégiés, où s'expérimentent et se nourrissent de façon effective, réelle, présente, les questions de la relation et de la mise en commun. Les ateliers obéissent, eux aussi, à cette conception.

A l'issue d'une séance que j'ai trouvée particulièrement féconde en février dernier, j'ai voulu dévoiler ce qui s'y jouait. Après le spectacle, nous étions une douzaine autour de la table, garnie de boissons et de quelques en-cas, dans la petite bibliothèque de $1^{\prime} \mathrm{ADC}^{23}$ : un cadre intimiste, convivial et chaleureux, propice à l'échange. Il y avait là trois ou quatre fidèles de la première heure (ayant participé aux six ateliers des saisons précédentes) mais

\footnotetext{
${ }^{22}$ Jacques Rancière, Le Spectateur émancipé, op. cit., pp. 28-29.

${ }^{23}$ Association pour la Danse Contemporaine à Genève : www.adc-geneve.ch [consulté le 26/06/2013].
} 
également de nouveaux participants avec, pour l'un d'eux, une première expérience en tant que spectateur de danse. Nous venions d'assister à Mash Up, le spectacle de Maud Liardon ${ }^{24}$.

Cette performance chorégraphique se propose comme une succession de sept tableaux subordonnés à un même principe de composition où il s'agit de créer un troisième élément en s'inspirant de deux éléments antérieurs. Ce principe musical, le Mash Up, donne son titre à la soirée ; à la soirée, et non à la pièce, tant - dès le début du débat - l'impression d'avoir affaire à un exercice de style s'est imposé à nous. Un premier tour de table fait état de remarques convergentes: certes la mise bout à bout des scènes obéit à des principes rythmiques (d'alternance entre la présence massive du rock et d'autres sources sonores, par exemple) mais personne n'y a décelé un argument fort renforçant la cohésion de l'ensemble. Je laisse s'installer ces premiers échanges sans intervenir car leur diversité met en évidence un manque d'homogénéité de la danse. Tout se passe comme si les interprètes, au fil des tableaux, semblaient changer de corps en même temps qu'ils changent de manière de danser. Un deuxième moment d'échange démontre que tous ont été attirés de diverses façons, par la sensibilité bien sûr, mais aussi par l'ironie, la révérence ou la poésie des propositions. Et si certains ont eu du mal à supporter ce bain sonore parfois violent «entrant dans nos têtes à coup de pointes », d'autres en revanche ont trouvé dans le plaisir de l'écoute du rock un prétexte à délaisser la chasse aux références...

A ce stade, et compte tenu du tour hétérogène que prend la discussion, je propose - en m'aidant du paperboard à ma disposition - de revenir de manière plus systématique sur chacun des moments chorégraphiques afin d'expliciter ces premières impressions diffuses.

En ouverture, et une dame signale que «à tout seigneur tout honneur!", la danse classique dévoile des corps aux bustes dénudés où la respiration et les articulations s'imposent là où, d'habitude, l'élégance trouve son terrain d'expression. Ici, j'interviens peu car le registre descriptif auquel j'ai habitué mes participants les plus anciens invite intuitivement les nouveaux à s'exprimer sur ce terrain. Parfois j'introduis un terme technique idoine en faisant remarquer, par exemple, que le vocabulaire chorégraphique employé multiplie les faillis, les relâchés qui font des danseuses des cygnes atypiques, tant du point de vue morphologique que technique. J'inscris deux références sur le tableaux dans la zone que je réserve à cet effet en bas à droite : celle de La Mort du Cygne de Fokine et la musique utilisée, signée des Black Angels, au nom lui aussi évocateur du vol. L’amateur de rock précise qu'il a trouvé la danse

${ }^{24}$ Un extrait du spectacle est visible à cette adresse : http://www.maudliardon.com/fr/video2 [consulté le 22/04/2013]. 
encore plus précaire et instable dans la violence sonore, faisant naître une grâce ultime presque par contraste.

L'allusion à la musique me permet de faire la transition avec la seconde partie. J'inscris au même endroit du tableau le Rid of Me de P. J. Harvey, que la chorégraphe a substitué à l'œuvre de Ravel pour sa version du Boléro de Béjart. Chacun a reconnu l'allusion à l'oeuvre (également popularisée par le film de Claude Lelouch Les Uns et les autres) : Béjart demeure bien le seul chorégraphe que peut citer tout néophyte en danse. Une discussion s'installe, un peu en marge du débat principal, sur le rôle de vulgarisateur de la danse tenu par Béjart dès les années 70 en France. Mais je recentre le débat sur l'intensité de la danse proposée. Une opposition se dessine sur la perte de la vitalité débridée du solo de référence, qui montre rétrospectivement - je le souligne au passage - combien la maîtrise des collectifs chez le chorégraphe français soutenait ses œuvres. Si tous les spectateurs ne partagent pas cet avis, chacun, en revanche, s'accorde à voir le chant faire irruption de façon très juste, ici comme dans toute la pièce, et venir sans cesse soutenir la danse.

Puis l'unanimité se fait à nouveau autour de la troisième séquence où la harpe parvient à dompter l'énergie grunge de Nirvana, que certains parmi nous ont d'ailleurs peiné à reconnaître! Plusieurs participants ont remarqué que les trois danseuses, par leur chant murmuré, parviennent à chasser la dimension racoleuse du cabaret que tous ont évoqué dès le départ. L'érotisme, qui rappelle à certains l'ambiance du film Ziegfeld Follies, s'estompe au profit d'une douce poésie. Les références continuent de s'accumuler en bas du tableau, les caractéristiques de la danse en haut à gauche. En dessous, je consigne les traits saillants qui se rapportent à la composition. Ainsi, je note quelques mots indiquant que les éventails palpent finement chaque parcelle d'espace en un délicat cocon où se réfugient les interprètes. $\mathrm{Ou}$ comment la densité légère des plumes fait écho à la promenade dentelée des doigts de la musicienne. Je réserve une troisième zone aux éléments scénographiques. Aux côtés des références et des données contextuelles qui sont évoquées dans le débat sont inscrits les partis pris chorégraphiques que nous avons relevés, notamment le parallèle entre mash up musical et son déplacement au chorégraphique. Ainsi se dessine, sur le tableau, un espace vide qu'entourent les 6 îlots évoqués et qui sont autant de portes d'entrées pour la prise de parole du spectateur. J'y tisse au fil des échanges un réseau de relations entre les éléments observés ; des flèches de couleurs variées se dessinent, s'épaississent ou s'effacent, s'annotent ou se transforment en hypothèses de lecture. Ainsi est figurée l'analyse en train de se faire et la manière dont le sens se construit collectivement. 
Pour nos deux amateurs de rock, spectateurs de danse débutants, la voix et l'énergie rock du danseur semble déstructurer encore davantage la danse empruntée à William Forsythe, qui - je le rappelle au passage - pousse toujours plus loin la révolution entamée par Balanchine sur les corps classiques. Dans cette partie, tout le monde s'accorde à voir la rencontre entre musique et danse comme frappée au sceau de l'évidence. Evidence que l'on retrouve dans la subtile déclinaison chromatique des costumes de la partie qui suit et nous invite à une lecture pop. Elle compense un malaise engendré par des corps diminués et comme contraints à la reptation, «comme si l'absence de musique laissait les corps amputés d'une part d'euxmêmes » ajoute encore pertinemment l'un de nos rockeurs. Ambiguïté vite dissipée par l'humour de salutations optimistes que s'adressent les danseurs et un Satisfaction (des Rolling Stones) hurlé à l'unisson et a capella. Dans la partie suivante, nombreux sont ceux qui ont détecté la référence au solo de Cyd Charisse dans Singin' in the Rain. Mais l'atmosphère fifties peine à convaincre. Le rapprochement semble ici inopérant pour créer le décalage espéré. En revanche la scansion des pulsations telluriques du Sacre du Printemps nous a évoqué à tous à la fois Pina Bausch et Nijinsky. Les voix et la gestuelle très rock rejoignent la dimension tragique de l'œuvre de Stravinsky. Hybride, la danse finie par oublier ses formes pour se sacrifier sur l'autel d'une énergie brute et débridée. Nous remarquons que ce dernier morceau est à l'image du spectacle tout entier, allant vers une libération de la vitalité irréductible des corps, signalant une dernière fois que la dimension kinesthésique qui nous envahit, tant en regardant la danse qu'en écoutant la musique, est toujours bien présente.

Nous avons dû, faute de temps, délaisser une lecture du genre dans le spectacle. Nombre d'indices s'y prêtaient pourtant, de l'attribution des instruments au changement de sexe de l'Elu(e) en passant par une dé-sexuation progressive de la danse, il y avait matière à investigation... mais je préfère recentrer le groupe, au moment de mettre un terme à l'atelier, sur une dimension que nous n'avons pas abordée et dont j'ai l'intuition qu'elle peut déboucher sur un consensus conclusif. En effet, la structuration de l'espace scénique par des lumières précises souligne judicieusement la danse de bout en bout. Cependant, nous nous sommes interrogés sur la partition du plateau entre danse et musique à partir d'un argument qui suggérait plutôt l'interaction. Cette rencontre à lieu, bien sûr, quand les danseurs s'approprient, parfois timidement, la gestuelle rock ; elle est surtout incarnée par une Maud Liardon omniprésente, et qui passe sans complexe des pointes à la batterie et à la basse, laissant son enthousiasme l'emporter sur sa technique instrumentale, ce que chacun a remarqué. Pour conclure la soirée, je fais observer que, ce faisant, la chorégraphe reste fidèle, sous des apparences actuelles, à un constant principe qui traverse le $\mathrm{XX}^{\circ}$ siècle, s'agissant du 
dialogue danse-musique : celui d'un art chorégraphique, demandant à un art musical qu'il juge plus savant que lui, la clé de sa composition. Maud Liardon ne déroge pas à cette règle mais si l'on veut, ce n'est là qu'un détail, et tous les participants abondent, car Mash Up nous est surtout apparu comme une invitation à faire jouer ses références avec intelligence, humour ou poésie; une archéologie de nos souvenirs que réactive la succession des scènes; une incitation à créer soi-même, de manière ludique et décomplexée. C'est aussi une façon de (re)découvrir les œuvres initiales, éclairées par ces « visitations ».

\section{Conclusion}

Cette étude de cas tente de rendre compte du déroulement d'un atelier tel que je les conçois. Il en reprend donc les principes de fonctionnement ainsi qu'une synthèse réécrite des échanges qui se sont instaurés au cours de cette session de février 2013. A l'écrit, il est difficile de rendre compte des interrogations et des dialogues - parfois digressifs mais souvent passionnants - sur les conceptions de l'art de chacun, ou encore les adresses que le spectacle a pu lancer à chaque spectateur participant. Il relate moins fidèlement qu'un verbatim les relations d'aide qui s'instaurent parfois entre spectateurs débutants et participants plus aguerris - ne fusse que le choix d'une expression juste, d'un éléments contextuel ou d'une référence à partager. Mais cette partie de l'article se veut trace de notre activité de ce soir-là, tout autant qu'invitation à comprendre ce qui se joue dans ces moments d'échange qui parfois intriguent aussi les équipes artistiques au sortir du plateau, qui se demandent ce que nous pouvons bien comploter à deux pas des loges, une fois le rideau baissé.

Je tiens beaucoup à cette dénomination $d^{\prime}$ '《Atelier» qui signale que, comme pour l'artiste, l'endroit est avant tout un lieu de production, fusse-t-il - dans notre cas - d'une simple parole. Mais celle-ci est essentielle à mes yeux. Elle permet de formuler collectivement des réponses aux questions que l'œuvre nous pose, sans injonction à produire dès la sortie de salle un simple, et souvent péremptoire, jugement de valeur (de «c'était génial » à « je n'ai rien compris ») ; jugement qui, au demeurant, ne dit rien de la pièce ni de notre réception sensible. L'atelier permet d'échapper au rythme souvent frénétique imposé par la vie sociale et culturelle. Il ose prendre son temps et ouvre une parenthèse dans l'immédiat après spectacle. Nul besoin d'une quelconque expertise car par le biais de la description et du débat, des hypothèses de lecture de l'œuvre se construisent sur l'écoute et l'échange, au gré des sensations, des rapprochements d'indices ou des souvenirs d'autres expériences. D'une durée souvent égale au spectacle lui-même (c'est-à-dire une à deux heures), il permet de s'inscrire dans une parenthèse temporelle qui autorise la redécouverte de sa propre sensibilité, 
comment elle permet aussi de poser sur un spectacle - quelquefois difficile d'accès - un regard bienveillant, et qui est éclairé par la mise en commun tout en s'enrichissant du «goût des autres ».

\section{Bibliographie}

Brousseau Guy, Théorie des situations didactiques, Grenoble, La Pensée sauvage, 1998.

Debray Régis, Vie et mort de l'image : une histoire du regard en Occident, Paris, Gallimard, coll. «Bibliothèque des idées », 1992.

Formis Barbara (ed.), Penser en corps. Soma-esthétique, art et philosophie, Paris, L'Harmattan, coll. «l'art en bref », 2009.

Maldiney Henri, Regard, Parole, Espace, Lausanne, L'Age d'homme, 1973.

Martel Frédéric, Mainstream, enquête sur cette culture qui plait à tout le monde, Paris, Flammarion, 2010.

Rancière Jacques, Le Spectateur émancipé, Paris, La fabrique, 2008.

- Le Partage du sensible, Paris, La Fabrique, 2000

- Le Maître ignorant. Cinq leçons sur l'émancipation intellectuelle, Paris, 10/18, coll. « Fait et cause », 2004.

Rochlitz Rainer, Le Vif de la critique, tome 2 : Esthétique et philosophie de l'art, Bruxelles, La Lettre volée, coll. « Essais », 2010.

Saez Jean-Pierre (ed.), Culture et société : un lien à recomposer, Toulouse, éd. de l'Attribut, 2008.

Simondon Gilbert, Du mode d'existence des objets techniques, Paris, Aubier, 1989.

Stiegler Bernard, De la misère symbolique 1. L'époque hyperindustrielle, Paris, Galilée, 2004.

- Aimer, s'aimer, nous aimer. Du 11 septembre au 21 avril, Paris, Galilée, 2003,

- «L'hyperindustrialisation de la culture et le temps des attrape-nigauds », in Internet all over, Art Press hors série, novembre 1999, 41-62. 